\title{
Nanostructured biocomposite films of high toughness based on native chitin nanofibers and chitosan
}

\author{
Ngesa E. Mushi ${ }^{1}$, Simon Utsel ${ }^{1}$ and Lars A. Berglund ${ }^{1,2 *}$ \\ ${ }^{1}$ Department of Fiber and Polymer Technology, Royal Institute of Technology, Stockholm, Sweden \\ ${ }^{2}$ Department of Fiber and Polymer Technology, Wallenberg Wood Science Center, Royal Institute of Technology, Stockholm, Sweden
}

\author{
Edited by: \\ Frederic Jacquemin, Université de \\ Nantes, France \\ Reviewed by: \\ Sylvain Freour, Institut de Recherche \\ en Génie Civil et Mécanique, France \\ Andrey Aniskevich, University of \\ Latvia, Latvia \\ *Correspondence: \\ Lars A. Berglund, Department of \\ Fiber and Polymer Technology, \\ Wallenberg Wood Science Center, \\ Royal Institute of Technology, \\ Teknikringen 56-58, SE-100 \\ 44 Stockholm, Sweden \\ e-mail: blund@kth.se
}

\begin{abstract}
Chitosan is widely used in films for packaging applications. Chitosan reinforcement by stiff particles or fibers is usually obtained at the expense of lowered ductility and toughness. Here, chitosan film reinforcement by a new type of native chitin nanofibers is reported. Films are prepared by casting from colloidal suspensions of chitin in dissolved chitosan. The nanocomposite films are chitin nanofiber networks in chitosan matrix. Characterization is carried out by dynamic light scattering, quartz crystal microbalance, field emission scanning electron microscopy, tensile tests and dynamic mechanical analysis. The polymer matrix nanocomposites were produced in volume fractions of 8,22 , and $56 \%$ chitin nanofibers. Favorable chitin-chitosan synergy for colloidal dispersion is demonstrated. Also, lowered moisture sorption is observed for the composites, probably due to the favorable chitin-chitosan interface. The highest toughness (area under stress-strain curve) was observed at 8 vol\% chitin content. The toughening mechanisms and the need for well-dispersed chitin nanofibers is discussed. Finally, desired structural characteristics of ductile chitin biocomposites are discussed.
\end{abstract}

Keywords: chitin nanofibers, chitosan, nanostructured, nanocomposites, mechanical properties

\section{INTRODUCTION}

Chitosan is a widely used biopolymer and interesting for use in packaging and biomedical applications. It is commercially available as a derivative of chitin microfibrils from crustaceans. The chitin molecule itself consists of $\mathrm{N}$-acetyl glucosamine units. The preparation of chitosan then involves derivatization through elimination of the chitin acetyl group, and the final sugar monomer is $\mathrm{N}$-glucosamine. In biological organisms, chitin is predominantly organized in extended chain conformation and assembled in the form of microfibrils (Figure 1). This structural organization is vital for the mechanical function of cuticles and exoskeletons of insects and crustaceans (Neville, 1967; Raabe et al., 2005). In addition, chitin structures provide support for tissues and organs such as muscles, eyes, throat etc. Chitosan is in nature less common, but is present as a cell wall component of filamentous fungi, where chitosan biosynthesis is through deacetylation of chitin (Bartnicki-Garcia, 1968; Muzzarelli et al., 2012).

Recently, chitin nanocrystals have been considered for nanocomposites (Gopalan Nair and Dufresne, 2003; Sriupayo et al., 2005; Mathew et al., 2009). Chitin nanowhiskers were combined with chitosan (Sriupayo et al., 2005; Shelma et al., 2008), polycaprolactone (Ji et al., 2012) or poly (vinyl alcohol) (Lee et al., 1996) to improve the mechanical properties of the polymer. One reason for the interest in chitin and chitosan is favorable wound healing properties (Yusof et al., 2003; Shelma et al., 2008; Murakami et al., 2010). However, mechanical properties of neat chitosan films leave room for improvement, as can be concluded from data in published studies; Young's modulus $E=2.4 \mathrm{GPa}$
(Ifuku et al., 2013), tensile strength $\sigma^{*}=40-100 \mathrm{MPa}$ (Mima et al., 1983; Park et al., 2002; Ifuku et al., 2013) and strain to failure $\varepsilon^{*}=6-100 \%$ (Mima et al., 1983; Park et al., 2002; Shelma et al., 2008; Fernandes et al., 2010). One may note the wide range in strain to failure due to differences in molar mass, environmental conditions and casting conditions. The mechanical properties of nanocomposites based on chitin nanowhiskers combined with polymer matrices such as chitosan (Sriupayo et al., 2005; Shelma et al., 2008), poly methylmethacrylate (Chen et al., 2014), poly (vinyl alcohol) (Lee et al., 1996) and polycarprolactone (Ji et al., 2012) have also been reported. The mechanical properties of these nanocomposites are generally low; strength $\sigma^{*}=84 \mathrm{MPa}$, strain to failure $\varepsilon^{*}=9 \%$ at $3 \%$ whisker content (Sriupayo et al., 2005); modulus $E=1.6 \mathrm{GPa}, \sigma^{*}=60 \mathrm{MPa}$ and $\varepsilon^{*}=7 \%$ at $17 \%$ whisker content (Shelma et al., 2008). Chemical cross-linking was also used to improve some mechanical properties of the nanocomposites; for example, chitin nanowhisker-chitosan scaffolds cross-linked via amine groups (Mathew et al., 2009). It is not clear why chitin nanowhisker reinforcement effects are so small, although there are several possible explanations. The aspect ratio is small with lengths in the range of $200-500 \mathrm{~nm}$ and diameters 6-20 nm (Yamamoto et al., 2010), the chitin content is often low and agglomeration effects may be present.

An interesting recent development is the extraction of chitin from crustaceans in the form of long nanofibers (Ifuku et al., 2010; Mushi et al., 2014a). The chemical and physical properties are very attractive; degree of acetylation $=87 \%$, diameter $=3-6 \mathrm{~nm}$, length $=800-1000 \mathrm{~nm}$. The aspect ratio (length/diameter) is in the same range as for cellulose nanofibers 


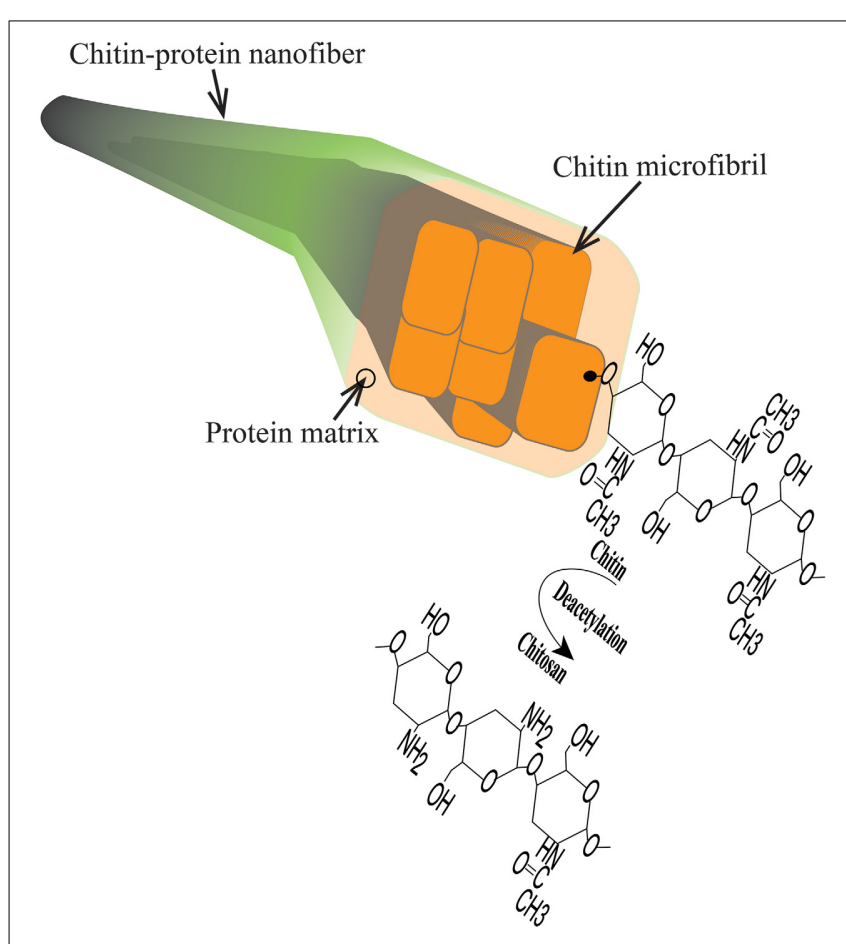

FIGURE 1 | Schematic diagram of chitin structures including chitinprotein nanofiber, chitin microfibril, chitin polymer chain and chitosan.

disintegrated from wood pulp (>100) (Henriksson et al., 2007). In preparation of chitin nanowhiskers (nanocrystals), treatment with concentrated $\mathrm{HCl}$ or $\mathrm{NaOH}$ leads to formation of shorter rods. In the present chitin nanofiber structure, the fibrous structure is much longer and possibly contain disordered regions. From a basic science point of view, it is interesting to compare with suspensions and polymer matrix nanocomposites based on fibrous nanocellulose. Effects from different structure and surface characteristics of fibrils as well as intrinsic fibril strength may be possible to estimate. In a more practical sense, chitin nanofibers can be used as a reinforcement phase in chitosan in order to improve the mechanical properties. Compared with chitin nanowhiskers, it may be possible to use higher chitin content and to better control the degree of fibril dispersion.

There are two major purposes of reinforcing chitosan-based films with nanofibers. First, to improve mechanical properties such as strength, modulus and toughness. For food packaging films, tensile strength above 50-70 MPa and high toughness are desirable (Chambi and Grosso, 2011). A second purpose is to reduce effects from the moisture affinity of chitosan. This include moisture sorption, swelling and reduced barrier properties. In the present study, focus is on mechanical properties. The first attempt to reinforce chitosan with high aspect ratio bio-nanofibers was in a well-cited study by Fernandes et al. (2010) where cellulose nanofibers were used. The nanocomposite films showed high optical transparency, a Young's modulus of about $6.8 \mathrm{GPa}$ and a strength of $115 \mathrm{MPa}$ at $60 \%$ volume fraction of cellulose (Fernandes et al., 2010). However, ductility was sacrificed. More recently, Ifuku et al. (2013) reported high strength (e.g., $\sigma^{*}=140 \mathrm{MPa}$ at $80 \mathrm{wt} . \%$ nanofiber content) for chitin-chitosan nanocomposites (Ifuku et al., 2013). The study employed a deacetylated chitin nanowhiskers/nanofibers concept. Chitin nanofibers with a deacetylated surface were combined with a chitosan matrix. The focus of the present study is to discuss strain to failure (in particular toughness) and deformation mechanisms. The ductility (strain to failure) of composites was observed to be lower as compared to neat chitosan-based films (Ifuku et al., 2013). The nanocomposite films based on deacetylated chitin nanofibers and chitosan showed slightly better modulus and strength $\left(E=7.8 \mathrm{GPa}\right.$ and $\sigma^{*}=125 \mathrm{MPa}$ at $60 \mathrm{wt} . \%$ nanofiber content, Ifuku et al., 2013) as compared to results from nanofibrillated cellulose and chitosan $(E=6.8 \mathrm{GPa}$ and $\sigma^{*}=115 \mathrm{MPa}$ at $60 \mathrm{vol} \%$ nanofiber content) (Fernandes et al., 2010).

In the present study, the possibilities to combine strength and ductility in order to obtain high work to fracture (area under stress strain curve) are in focus. The chitin nanofibers are different from those reported in earlier studies (Ifuku et al., 2013). The present origin is lobster rather than crab, and the present nanofibers have lower protein content and higher degree of acetylation, see Mushi et al. (2014a). The present study discusses the importance of the colloidal state and suggests routes toward material compositions and nanostructures with even higher toughness, based on observed deformation behavior. We emphasize the importance of colloidal stability and report low composite moisture sorption due to the favorable chitin/chitosan interface. The resulting nanocomposites show considerable ductility and toughness. This is related to the intrinsic chitosan ductility and the well-dispersed nanostructured network of chitin nanofibers in the final material. The chemical chitin-chitosan compatibility is also an important factor. The use of chitosan allows for compositional tailoring (chitin content and degree of acetylation) to meet requirements in a variety of packaging or wound healing applications.

\section{MATERIALS AND METHODS MATERIALS}

Low protein native chitin nanofibers were disintegrated from lobster Homarus Americanus of Northwest Atlantic, produced in Canada, according to the procedure reported in our previous work (Mushi et al., 2014a). The lobster was cleaned to take away salts and tissues. Demineralization to remove calcium carbonate minerals was performed with $2 \mathrm{M} \mathrm{HCl}$ twice for a duration of $1 \mathrm{~h}$ in each step. In the first step, treatment was done on large exoskeleton pieces to reduce dust from mineral particles during grinding. The sample was freeze dried. It was crushed with a $500 \mu \mathrm{m}$ mesh size (Retsch grinder, Model ZM200, Germany) to produce crude chitin powder. Second, demineralization was performed on the freeze dried crude chitin powder. Depigmentation was followed by washing for $12 \mathrm{~h}$ overnight with ethanol (96\%). Lastly, protein was removed by treatment with $20 \% \mathrm{NaOH}$ for 2 weeks. The chitin sample was washed in deionized water between each step. Another washing step was performed with $4 \%$ acetic acid until the suspension of chitin powder turned whitish. The white creamy suspension of chitin powder was mechanically treated in a blender (VM0105E, USA). It was homogenized 
through a Microfluidizer (M-110EH, Microfluidics Ind., Newton, MA, USA) so that a translucent hydrocolloid of chitin nanofibers was obtained. Degree of acetylation, DA, ranged between 86 and $87 \%$ based solid state ${ }^{13} \mathrm{C}$ NMR (Nuclear Magnetic Resonance Spectroscope). Chitosan powder from shrimp (high viscous, Sigma, Germany) with a degree of acetylation of less than $15 \%$ was used. It was dissolved in acetic acid (1.0 wt.\%), and aggregates where removed by centrifugation $(4000 \mathrm{rpm}, 10 \mathrm{~min}$, room temperature).

\section{DYNAMIC LIGHT SCATTERING (DLS)}

The zeta potential $(\zeta)$ and aggregate size of the chitin nanofiber hydrocolloid was studied by dynamic light scattering using Zetasizer Nano, Model ZEN3600 (Malvern Instruments Ltd., UK). The light source was operated at a wavelength of $633 \mathrm{~nm}$. The chitin nanofiber suspension was diluted to a concentration of $50 \mathrm{mg} / \mathrm{L}$ at $\mathrm{pH} 3$ and filled in a PMMA (Poly Methyl Methacrylate) cuvette and scanned three times at ambient conditions (i.e., $25^{\circ} \mathrm{C}$ ).

\section{QUARTZ CRYSTAL MICROBALANCE (OCM)}

A Quartz Crystal Microbalance Model QCM-E4 from Q-Sense AB (Västra Frölunda, Sweden) was used to study chitosan adsorption to a chitin nanofiber surface with a continuous flow of $100 \mu \mathrm{L} / \mathrm{min}$ (Marx, 2003). The crystals were AT-cut quartz crystals with a $5 \mathrm{MHz}$ resonance frequency and an active surface of sputtered silica. These were rinsed with Milli-Q water, ethanol and Milli-Q water, dried in nitrogen, and then placed in an air plasma cleaner (Model PDC 002, Harrick Scientific Corporation, NY, USA) under reduced air pressure for $120 \mathrm{~s}$ and $30 \mathrm{~W}$. A $1 \mathrm{~g} / \mathrm{L}$ chitin nanofiber suspension was spin-coated on the cleaned crystals resulting in a fully covered chitin nanofiber surface. The change in frequency can be used to estimate the change in adsorbed mass according to the Sauerbrey model Equation (1) (Sauerbrey, 1959).

$$
m=C \times \triangle f / n
$$

where, $m$ is the adsorbed mass per unit area $\left(\mathrm{mg} / \mathrm{m}^{2}\right), C$, the sensitivity constant $=-0.177\left[\mathrm{mg} /\left(\mathrm{m}^{2} \cdot \mathrm{Hz}\right)\right], \Delta f$, the change in resonant frequency $(\mathrm{Hz})$, and $n$ is the overtone number.

\section{PREPARATION OF NANOSTRUCTURED COMPOSITES}

A colloidal suspension of ca. $1 \mathrm{wt} \%$ solid content of chitin nanofibers and a chitosan solution in at least $4 \%$ acetic acid (initially the concentration of acetic acid was $1 \mathrm{wt} . \%)$ was slowly mixed under magnetic stirring for $12 \mathrm{~h}$ overnight to allow a uniform mixture. Casting was done on a Teflon film surface securely clamped to a glass cylinder with a diameter of $72 \mathrm{~cm}$. This technique was employed for the preparation of nanopaper membranes in previous studies (Henriksson et al., 2008; Sehaqui et al., 2010; Mushi et al., 2014b). Pure chitin films were sensitive to moisture, so controlled drying of the composite film was performed in the presence of excess acetic acid and low temperature condition in an oven at $37^{\circ} \mathrm{C}$ in order to avoid warpage or uneven distribution of chitosan and nanofibers in the solid film. Evaporation of water and acetic acid resulted into a consolidated nanostructured composite film. Several films of the same volume fraction were prepared, and at least two were used for each composition in this study. The previously established nanopaper preparation procedure was employed for the preparation of nanopaper membranes (Sehaqui et al., 2010; Mushi et al., 2014b).

\section{STRUCTURAL CHARACTERIZATION}

Structural characterization of the nanostructured composite was performed in a Field Emission Scanning Electron Microscope (FE-SEM) S-4800 (Hitachi). The sample was conditioned in a desiccator for $12 \mathrm{~h}$ overnight to remove moisture and then platinum-palladium sputtered in Agar HR Sputter Coater prior to structural imaging in the SEM. Surfaces of the nanostructured chitin membrane and composite were studied and a secondary electron detector was employed for capturing images at $1 \mathrm{kV}$. Porosity determination was based on the density method reported in previous work (Mushi et al., 2014b). Void content, $V_{v}$, was deduced from Equation 2. Equation 3 is the theoretical density, $\rho_{c}$, of void-free composite used in earlier work (Sehaqui et al., 2011). Weight fraction, $W$, was related to volume fraction, $V$, based on Equation 4. The subscripts stand for; $v$ - voids, $c$ void-free composite, $f$ - chitin nanofiber, sample refers to the real composite with voids and chitosan is the real matrix without voids. The measured density of chitosan film was considered as a true density of chitosan, $\rho_{\text {chitosan }}$. The density of dry chitin, $\rho_{f}$, is $1.425 \mathrm{~g} / \mathrm{cm}^{3}$ according to literature (Carlström, 1957).

$$
\begin{aligned}
V_{v} & =1-\frac{\rho_{\text {sample }}}{\rho_{c}} \\
\rho_{c} & =\frac{1}{\frac{W_{f}}{\rho_{f}}+\frac{\left(1-W_{f}\right)}{\rho_{\text {chitosan }}}} \\
V_{f} & =\frac{W_{f}}{\rho_{f}} \times \rho_{\text {sample }}
\end{aligned}
$$

\section{MECHANICAL CHARACTERIZATION}

Tensile tests were performed using Instron Universal Tensile Testing Machine Model 5944 (UK) equipped with a $500 \mathrm{~N}$ load cell. The specimens were conditioned in a room with $50 \%$ relative humidity and $23^{\circ} \mathrm{C}$ for $12 \mathrm{~h}$ overnight. For each volume fraction, at least five specimens were prepared with width and length of $5-40 \mathrm{~mm}$, respectively. Sample thicknesses were typically $60-80 \mu \mathrm{m}$. Tensile tests were performed at a strain rate of $4 \mathrm{~mm}$ per min. Mechanical properties such as tensile modulus, $\mathrm{E}$, tensile strength, $\sigma^{*}$, tensile strain to failure, $\varepsilon$ and work to fracture, $\mathrm{U}$ were estimated based on conventional analysis of nominal stress-strain curves. Tensile samples were conditioned at 50 and $90 \%$ relative humidy $(\mathrm{RH})$ and weighed to analyze the effect of moisture absorption in relation to mechanical behavior. Relative humidity was controlled in a dessicator with various salts and weight change was calculated from ratio of weight before and after saturation in high relative humidity, according to description in previous work (Mushi et al., 2014b). Dynamic mechanical analysis (DMA) was performed in TA Instruments equipment (Model Q800). In this equipment, dynamic heating ranged from -100 to $300^{\circ} \mathrm{C}$ at a rate of $3^{\circ} \mathrm{C} / \mathrm{min}$ and a frequency of $1 \mathrm{~Hz}$, change in storage modulus and $\tan \delta$ was recorded. Samples 
(width $=5 \mathrm{~mm}$, length $=10 \mathrm{~mm}$ ) were conditioned at $80^{\circ} \mathrm{C}$ for $10 \mathrm{~min}$ in order to stabilize moisture content.

\section{RESULTS AND DISCUSSION \\ CHITIN NANOFIBER COLLOID IN CHITOSAN SOLUTION}

Disintegration of the lobster exoskeleton was successfully performed according to procedure in the experimental section. A viscous transluscent hydrocolloid was obtained at $\mathrm{pH} 3$ in the presence of acetic acid. The structure and composition of the chitin nanofibers were previously reported (Mushi et al., 2014a). Briefly, the average protein content was 4.7 , with $95.3 \%$ chitin (Mushi et al., 2014a). In the present study, the nanofibers can be described as semiflexible fibrils with an average diameter of $10 \mathrm{~nm}$ and an average length of $1 \mu \mathrm{m}$. Note that in the biology community, the smallest fibrils are often termed microfibrils. Chitin microfibrils are 3-4 $\mathrm{nm}$ in diameter and embedded in proteins to form larger diameter, fibrous chitin aggregates (Raabe et al., 2006; Mushi et al., 2014b). "The present” nanofibers illustrated in Figure 1 are aggregates of several microfibrils and much reduced protein content compared with the native structure. It is likely that the surface of the nanofibers are chitin-rich with some deacetylation, see Figure 1. The chitosan polymer was obtained from Sigma Aldrich, and is a chitin derivative prepared by dissolution and deacetylation of chitin from shrimp exoskeletons. The chitin nanofiber colloid was mixed with the chitosan solution, See Materials and Methods Section.

The zeta potential and aggregate size of the colloidal chitinchitosan mixture were estimated for different composites based on DLS data. Zeta potential is an electrokinetic potential between the interfacial double layer of the chitin nanofiber and a reference point in the bulk liquid. Particle size estimations are also based on diffusion rate (Fall, 2013). Table 1 presents the zeta potential and chitin aggregate size data for the chitin nanofiber suspension at different concentrations of chitosan. In Figure 2, the size distribution estimates based on DLS are presented, (A) pure chitin colloid and pure chitosan solution and in (B) the chitin/chitosan colloidal mixtures. Note that the "size" can only be interpreted as a relative measure at this stage. The zeta potential data are in agreement with previous results by Fan et al. (2012). As an estimate, the threshold value for a stable colloid is $\geq+30$ or $\leq-30 \mathrm{mV}$. If we compare Figure 2A and Figure 2B, the most apparent effect is that the chitin aggregate "size" decreased from 634 to $165 \mathrm{~nm}$ after mechanical mixing with the chitosan solution. The charged chitosan molecules are able to reduce the size of chitin aggregates.

Table 1 | Zeta potential $(\zeta)$ and nominal chitin aggregate size $(\mathrm{nm})$ of chitin nanofiber hydrocolloids in chitosan solution with composition expressed as weight fraction.

\begin{tabular}{lccccc}
\hline Sample description (Chitin wt. \%) & 100 & 70 & 30 & 10 & 0 \\
Zeta potential (mV) & +45 & +65 & +64 & +58 & +45 \\
Average particle size (nm) & 670 & 295 & 190 & 220 & 220
\end{tabular}

Note that particle size is a relative estimate for comparative purposes, since the nanofibers have non-ideal geometry. The dry content was $0.005 w t \%$. The hydrocolloid contains about $4 \%$ acetic acid, and measurements were done at $\mathrm{pH} 3$.
The peak at lowest particle size for chitin colloids is believed to originate from the wide distribution in chitin fibril size. Note also that the chitosan solution shows large particles, Figure 2A, which indicate the presence of chitosan agglomerates rather than an ideal solution. In the chitin/chitosan mixtures, Figure 2B, those chitosan agglomerates are no longer present. Also, the small size peak for neat chitin is not present. The DLS data confirm the visible impression that the present chitin-chitosan colloid mixture forms a stable colloidal suspension. This is important since a prerequisite for well-dispersed chitin nanofibers in the solid nanocomposite material is well-dispersed chitin also in the colloid. The data can be compared with zeta potential data for stable TEMPO-oxidized cellulose nanofiber hydrocolloids (from -39 to $-52 \mathrm{mV}$ ) (Fall et al., 2011; Fall, 2013). The reason for the positive charge on the chitin nanofibers is partial deacetylation, which results in partially chitosan-like nanofiber surfaces.

The combination of chitin nanofibers with chitosan is of specific interest because of the potential for high compatibility (strong molecular interactions) at the nanofiber-polymer matrix interface. The chitin/chitosan charge repulsion in the colloid is apparently positive in that chitin agglomerate size is reduced, see Figure 2. Figure 3 presents QCM results of chitin nanofiber and chitosan mixtures at $\mathrm{pH} 3$. A spin-coated chitin nanofiber model surface was exposed to a chitosan solution $(100 \mathrm{mg} / \mathrm{L})$ in acetic

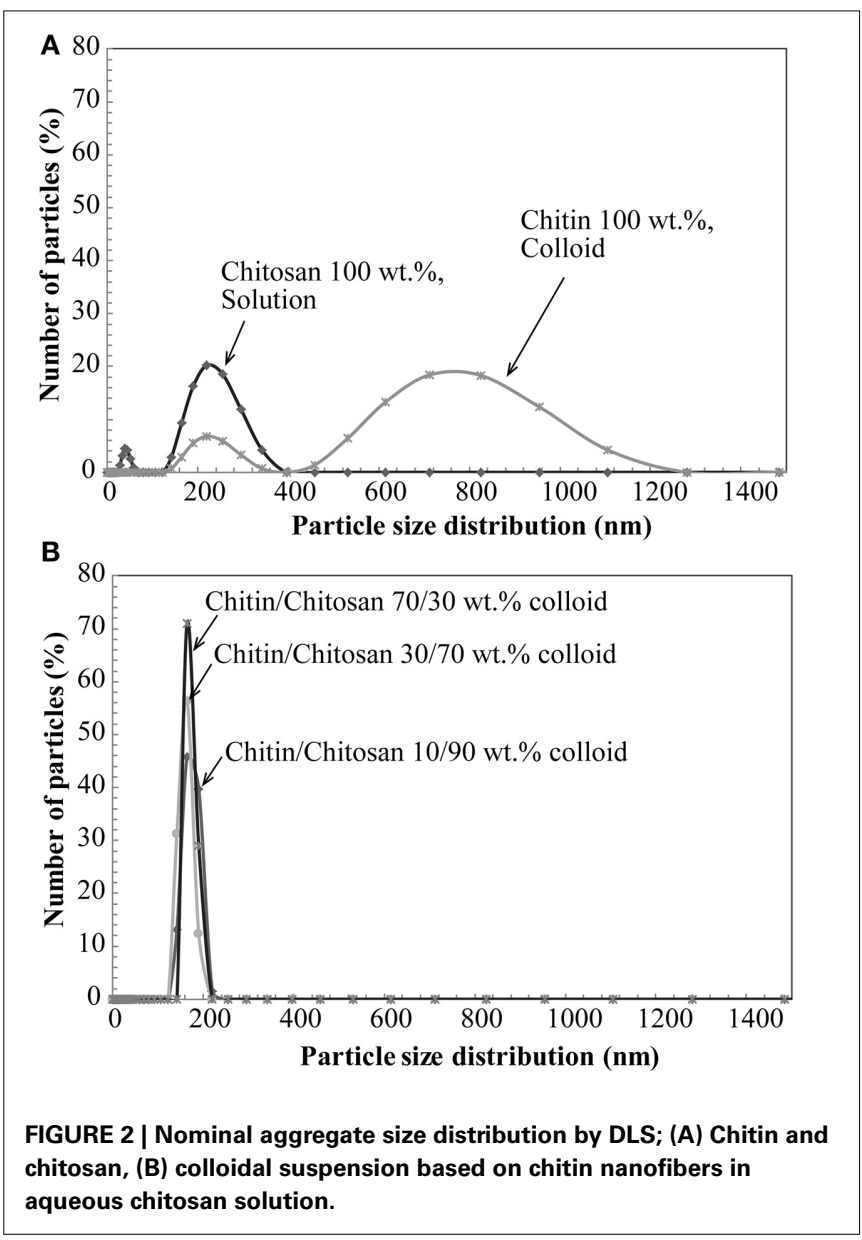


acid for $30 \mathrm{~min}$. The baseline was first established with the neat acetic acid solution. The QCM curve in Figure 3 shows no change in baseline with the addition of chitosan during the washing and rinsing steps, and it is concluded that no chitosan is adsorbed. This confirms the charge repulsion phenomenon in the colloid between the chitosan and the chitin nanofiber surface at $\mathrm{pH} 3$.

Due to deacetylation, there is a large concentration of amine groups in the present chitosan (above $85 \%$ of the maximum possible content). Although bulk degree of acetylation (DA) in our native chitin nanofibers was between 86 and $87 \%$, the nanofiber surface is much more deacetylated compared with the core. A degree of deacetylation of about $50 \%$ was estimated in a previous study (Das et al., 2012). The QCM results in Figure 3 thus correlate well with DLS data and the stable behavior of the chitinchitosan colloidal mixture. The chitosan did not adsorb to chitin nanofibers due to electrostatic repulsion.

\section{PREPARATION OF CHITIN-CHITOSAN NANOCOMPOSITES}

The nanostructured composite film was prepared by a simple film casting procedure where the liquid phase was evaporated. Table 2 presents densities and porosities of the composite films, as well as neat chitosan film and chitin membrane. With the exception of the non-porous chitosan films, porosities are estimated to be in the $13-20 \%$ range. In the context of physical properties, the volume fraction of reinforcement is the physically correct parameter, and Table 2 shows a significant difference between weight fraction and volume fractions due to the lower density of chitosan compared with chitin. The highest volume fraction of chitin is

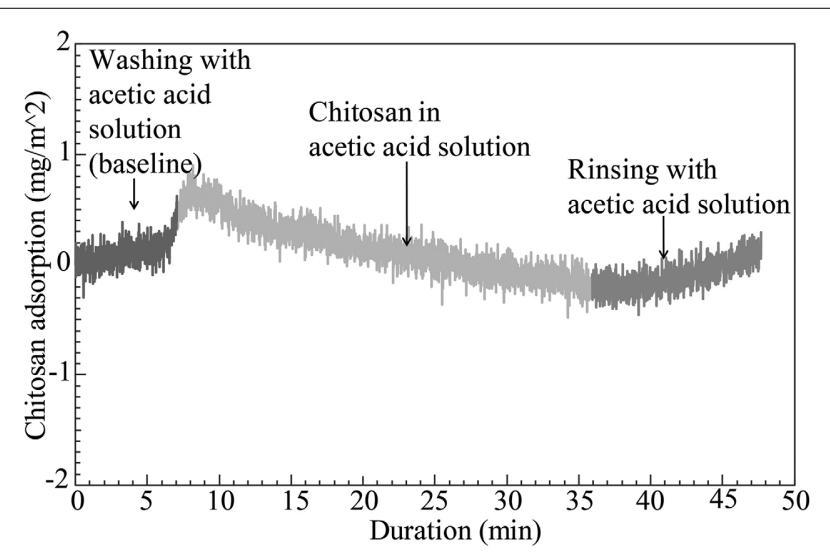

FIGURE 3 | QCM curve for characterization of chitosan adsorption on spin-coated chitin nanofiber model surface exposed to a chitosan solution at pH 3.

Table 2 | Data for density and porosity: Neat chitosan (0 wt. \% chitin), chitin/chitosan composites, and neat chitin porous membrane (100 wt. \% chitin).

Chitin nanofiber weight fraction (\%)

Chitin nanofiber volume fraction (\%)

Density of sample with voids $\left(\mathrm{g} / \mathrm{cm}^{3}\right)$

Density of void-free composite $\left(\mathrm{g} / \mathrm{cm}^{3}\right)$

Porosity (\%)

$\begin{array}{ccccc}0 & 10 & 30 & 70 & 100 \\ 0 & 8 & 22 & 56 & 84 \\ 1.22 & 1.08 & 1.03 & 1.13 & 1.21 \\ 1.22 & 1.24 & 1.28 & 1.36 & 1.425 \\ 0 & 13 & 20 & 17 & 16\end{array}$

$56 \%$ with the present preparation procedure and this provides potential for strong property enhancements.

The purpose was to study ductility of an all-chitin-based composite based on chitin nanofibers in a chitosan matrix. The liquid phase is the water-acetic acid mixture. Slow evaporation was carried out in order to reduce warpage from concentration gradients of water-acetic acid. The state of swelling in a local region depends on water-acetic acid concentration, so that large through-thickness differences in concentration and swelling strains can cause warpage. The effect of acetic acid on chitosan may show similarities to the effect of glycerol on starch films. The presence of acetic acid was reported to induce conformational changes in chitosan conformations (Kienzle-Sterzer et al., 1982), so that the ductility was improved.

\section{STRUCTURAL CHARACTERIZATION}

The FE-SEM micrograph in Figure 4A presents the upper surface of a porous neat chitin nanofiber membrane. The nanofiber population contains both small nanofibers with diameters at a scale of ten $\mathrm{nm}$, as well as larger agglomerated nanofiber bundles with diameters at the $100 \mathrm{~nm}$ scale. The nanofibers have curved geometries primarily random in-plane and to some extent out-of-plane. Pores at a typical scale of $10-40 \mathrm{~nm}$ are apparent as dark regions and there is considerable surface roughness. Figure 4B is the surface of the nanostructured chitin-chitosan matrix composite. The chitin nanofiber network is still apparent at a chitin volume fraction of $56 \%$. According to data in Table 2, the bulk porosities are comparable (17\% in B and $16 \%$ in $\mathbf{A}$ ) and pores are visually apparent in Figure 4. The chitosan matrix in Figure 4B appears to be well distributed.

\section{UNIAXIAL TENSILE PROPERTIES}

Figure 5 presents the stress-strain curve of the nanostructured chitin membrane $\left(V_{f}=84 \%\right)$ and the nanostructured composites $\left(V_{f}=8,22,56 \%\right)$ as well as data for neat chitosan films. The most important observation is that the nanostructured composite $\left(V_{f}=8,22,56 \%\right)$ shows high strain-to-failure for all compositions. Strain-hardening is observed in the post-yield region for $84,56,22$, and $8 \%$. For the other materials, there is initial strain-softening, followed by strain-hardening associated with chitin nanofiber network reorientation. The behavior is analogous to cellulose nanofiber composites (Sehaqui et al., 2011). However, at 8 and $22 \%$ chitin volume fraction, two plastic deformation regions are apparent. In a previous study of cellulose nanofibers in hydroxyethyl cellulose matrix, the second plateau was assigned to plastic deformation in matrix-rich regions between nanofiber-rich lamellae (Sehaqui et al., 2011). The 8\% composition is interesting. The yield strength (stress at onset of non-linear behavior) increases strongly compared with the neat chitosan (from 32 to $51 \mathrm{MPa}$, see Table 3). Most likely, composite yielding is associated with onset of chitosan shear yielding. The global yield stress is strongly increased for composites due to the load-carrying capability (stiffness) of the chitin nanofiber network (local chitosan stress becomes much lower than the global composite stress). In addition, the strain-to-failure is even higher than for neat chitosan. One may speculate that failure is associated with growth of nanoscale voids, and this process is delayed to 


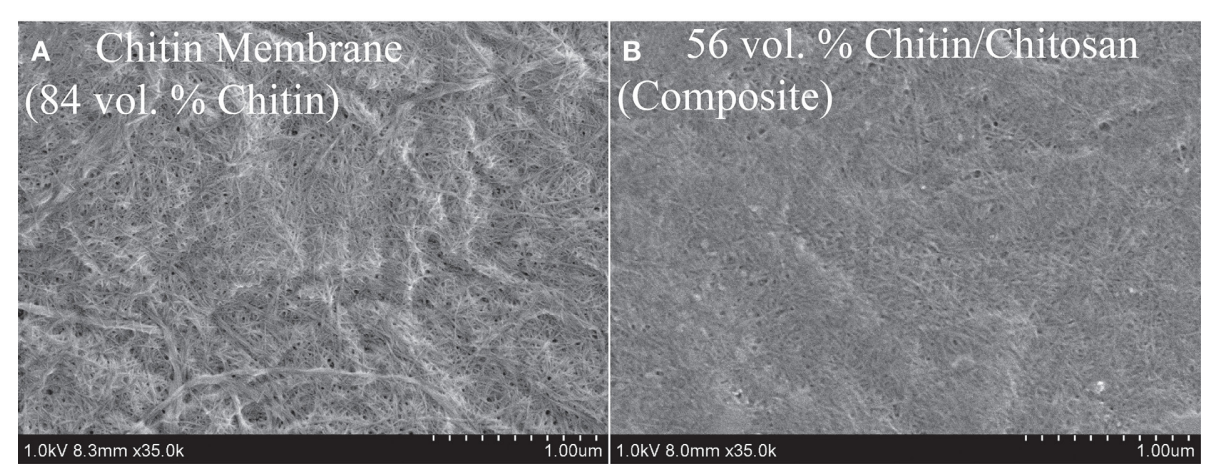

FIGURE 4 | SEM topographical view of (A) 84 vol.\% porous neat chitin membrane, and (B) 56 vol.\% chitin/chitosan nanocomposite (also porous).

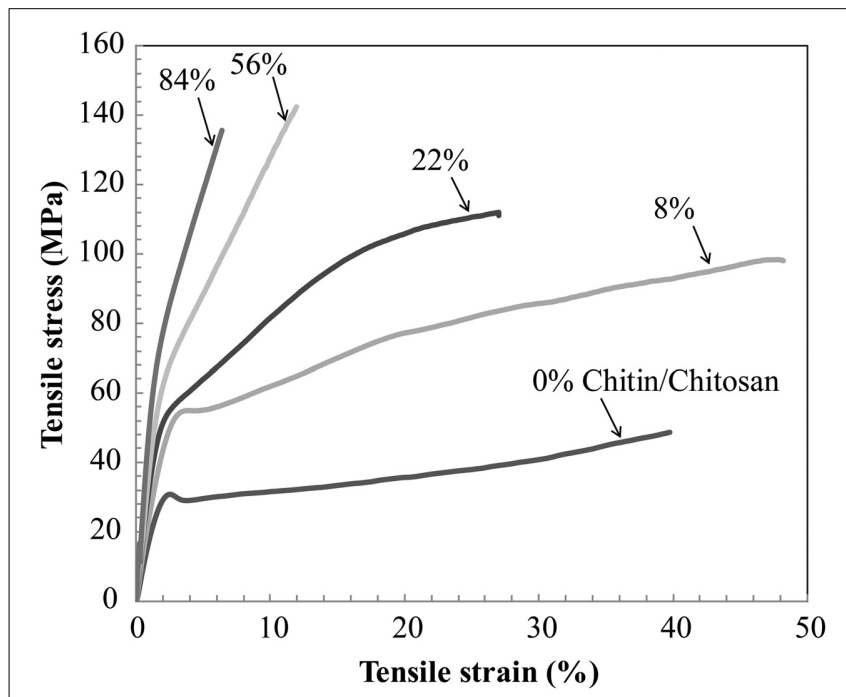

FIGURE 5 | Uniaxial tensile stress-strain curves of nanostructured composites and reference materials. $V_{f}$ stands for volume fraction of chitin nanofibers.

higher strains due to the presence of the chitin nanofiber network. For the $V_{f}=22 \%$ composition, strain to failure is decreased compared with $V_{f}=8 \%$. One may note that for $V_{f}=22 \%$, the stress level is much higher at a given strain in the plastic region, and this is likely to cause failure at lower strain. Some of the chitin nanofibers are subjected to very high local stress, which is much higher than the average nanocomposite global stress. This will result in local chitin nanofiber fracture and lowered strain to failure. For the nanostructured neat chitin membrane, the stress-strain curve shows yielding associated with inter-nanofiber separation dominated by opening tension or shear stresses at the local scale. Then follows substantial strain-hardening associated with nanofiber reorientation and interfibril slippage. This behavior has been discussed in previous studies on cellulose and chitin nanofiber membranes (Svagan et al., 2007; Henriksson et al., 2008; Sehaqui et al., 2011; Mushi et al., 2014a). Table 3 summarizes the mechanical properties of the present materials. The observed nanocomposite ductility is very large, and due to the
Table 3 | Tensile properties of nanostructured composites and reference materials (nanostructured neat chitin membrane and neat chitosan film; $84 \%$ means neat chitin membrane with $16 \%$ porosity, $0 \%$ means neat chitosan).

\begin{tabular}{|c|c|c|c|c|c|}
\hline Chitin content (vol.\%) & 0 & 8 & 22 & 56 & 84 \\
\hline Young's modulus (GPa) & $2.2(0.2)$ & $3.2(0.6)$ & $4.3(0.2)$ & $5.4(0.7)$ & $7.3(0.4)$ \\
\hline Tensile strength (MPa) & $52(5)$ & $98(3)$ & $114(3)$ & $141(3)$ & $153(11)$ \\
\hline Yield strength (MPa) & $32(1)$ & $51(3)$ & $53(2)$ & $63(4)$ & $70(2)$ \\
\hline Tensile strain to failure (\%) & $42(2)$ & $46(4)$ & $24(5)$ & $11(1)$ & $8(1)$ \\
\hline Work to fracture $\left(\mathrm{MJm}^{-3}\right)$ & $16(0.2)$ & $35(2)$ & $22(3)$ & $12(0.2)$ & $8(0.2)$ \\
\hline
\end{tabular}

The numbers in bracket are standard deviation.

strain-hardening behavior, the work to fracture (defined as the as the area under the stress-strain curve) also becomes very high. It simply means that substantial mechanical energy is required to cause final fracture. The highest work to fracture values are obtained for the 8 and 22 vol.\% chitin compositions.

Figure 6 shows SEM fracture micrographs of a nanostructured composite $\left(V_{f}=8 \%\right)$ and the nanostructured neat chitin membrane. Figure 6A is a topographical image of the nanocomposite film surface at $0 \%$ strain. The comparable smoothness of this surface corresponds to the high chitosan content. The estimated small-scale porosity is still substantial (13\%). The micrograph in Figure 6B shows the film surface close to the fracture plane at $45 \%$ strain after mechanical testing. Substantial chitin nanofiber reorientation is apparent so that the nanofibers are preferably in the direction of uniaxial loading. Figures $6 \mathrm{C}, \mathrm{D}$ present the cross-sectional fracture surfaces. Chitin fibrils are observed as fine protrusions on the fractured surface. Figure $\mathbf{6 C}$ shows the nanostructured chitin membrane and Figure 6D the nanocomposite $\left(V_{f}=8 \%\right)$. For the nanostructured membrane, although the structure appears layered, this layering is less distinct than for cellulose nanopaper (Henriksson et al., 2008). The fracture surface is rough, and there are indications of fracture and pull-out of layers from adhering layer neighbors. For the nanocomposite in Figure 6D, the fracture surface is more smooth, and the apparent fracture surface layering indicate that layer fracture is important. Fractured chitin nanofibers with diameters at the scale of tens of nanometers are apparent, although the nanofiber pullout lengths are very short. Signs of substantial matrix plasticity 


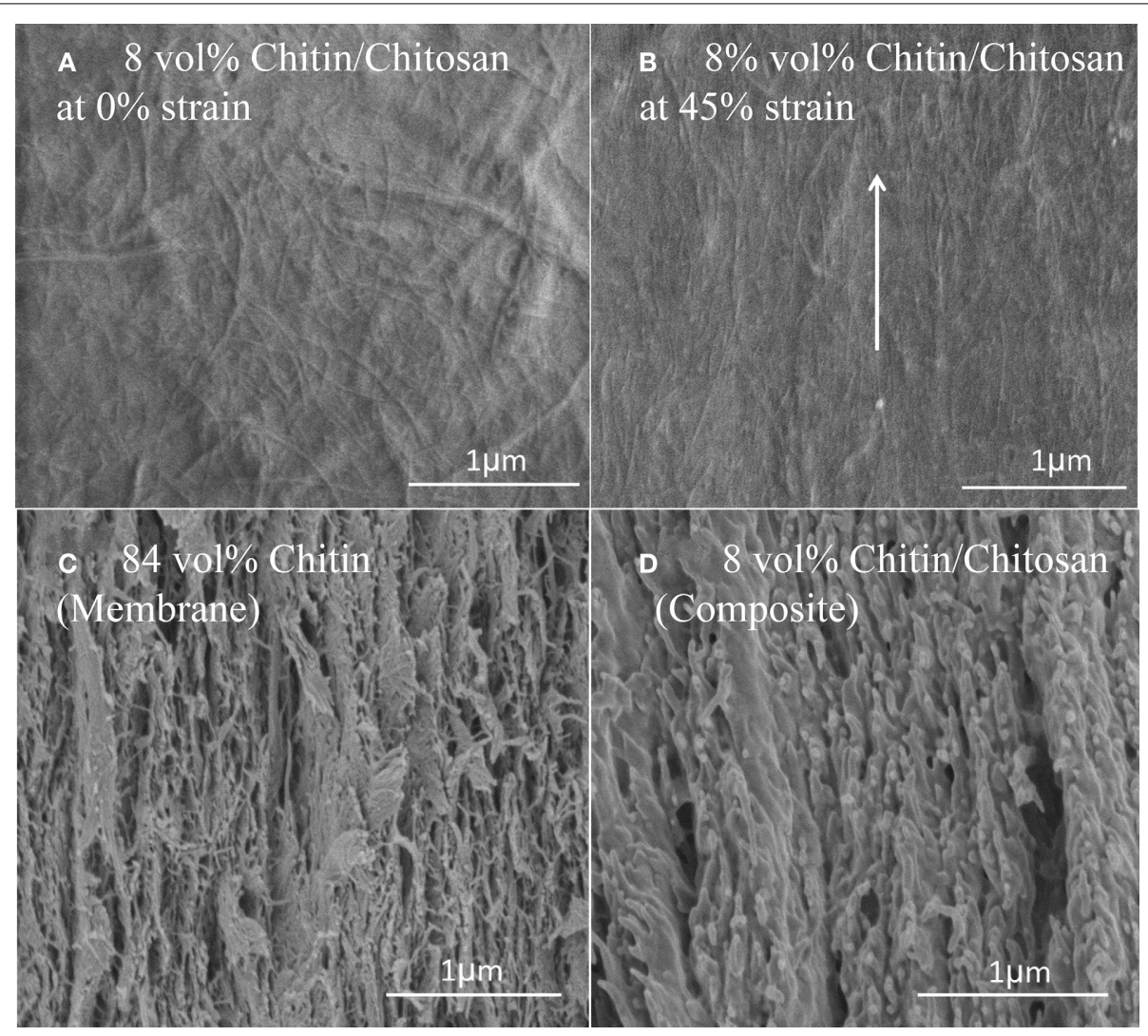

FIGURE 6 | FE-SEM micrographs of the $\boldsymbol{V}_{\boldsymbol{f}}=\mathbf{8} \%$ chitin composite surface: (A) at $\mathbf{0} \%$ strain (B) at $\mathbf{4 5} \%$ strain-to-failure. Fractured cross section FE-SEM micrographs (C) $V_{f}=84 \%$ chitin (D) $V_{f}=8 \%$ chitin. The arrow indicates loading direction and major fibril orientation direction after deformation.

are apparent in the smooth lamellae surfaces. There are similarities with fracture surfaces in cellulose nanofiber composites with plasticized starch matrix in terms of layered structure, fractured fibers of short pull-out lengths, plastic deformation features of the matrix (Svagan et al., 2007) and reorientation of nanofibers (Sehaqui et al., 2012).

From Table 3, it was observed that as chitin volume fraction increases, the modulus and strength are increased. In Figures $\mathbf{7 A - C}$, tensile modulus, strength and work to fracture for chitin materials are plotted as a function of chitin volume fraction. There is relatively stronger property increase at lower volume fraction. For a given fiber orientation distribution, tensile modulus depends on intrinsic modulus of constituents and the fiber volume fraction. There is a relatively weaker reinforcement effect at higher volume fractions, possibly due to chitin agglomeration. If chitin is present in the form of localized porous floc network entities, the reinforcement efficiency will be lower than for individually dispersed nanofibers in a polymer matrix. Toughness expressed as "work to fracture", the area under the stress-strain curve is as high as $35 \mathrm{MJ} / \mathrm{m}^{3}$ with $46 \%$ strain to failure at a volume fraction of $8 \%$ chitin. The use of acetic acid is important, since it can improve solubility of chitosan in water. The strength of chitosan-based films have been reported to depend on acetic acid content and solvent type (Park et al., 1999, 2002) but also degree of acetylation and chitosan molar mass
(Mima et al., 1983). Higher solubility leads to more favorable chitosan conformations in the solid composite film and correspondingly higher strength. Higher molar mass also increases strength through increased effects from physical entanglements of chitosan molecules. According to Park et al. (2002), tensile strength and strain to failure of chitosan films increased from 69 to $150 \mathrm{MPa}$ and $4.1-76 \%$, respectively, when $2 \%$ acetic acid was added. Again, the most likely reason is improved chitosan solubility and more favorable chitosan-chitosan mixing as well as more favorable chitosan conformations in the film. Chitin nanofiber colloidal properties also depend on molecular interactions (Qi et al., 2013) and this influences the degree of dispersion and the nanostructural details of the film. Poor dispersion in the collolid leads to agglomerate formation which may act as defects in the film so that the strain to failure is decreased.

Moisture sorption data are presented in Table 4. It is interesting to note that the chitin/chitosan nanocomposites show lower moisture content than neat chitosan as well as neat chitin nanofiber membranes. For chitin moisture sorption, the chitin nanofiber surface is the main site for water molecule sorption. The question is then how a chitosan matrix can reduce chitin-related moisture sorption. If hydroxyls and other sites at the chitin microfibril surface are interacting strongly with the chitosan matrix, potential sites for water molecules become occupied. As a consequence, the total moisture sorption of the 


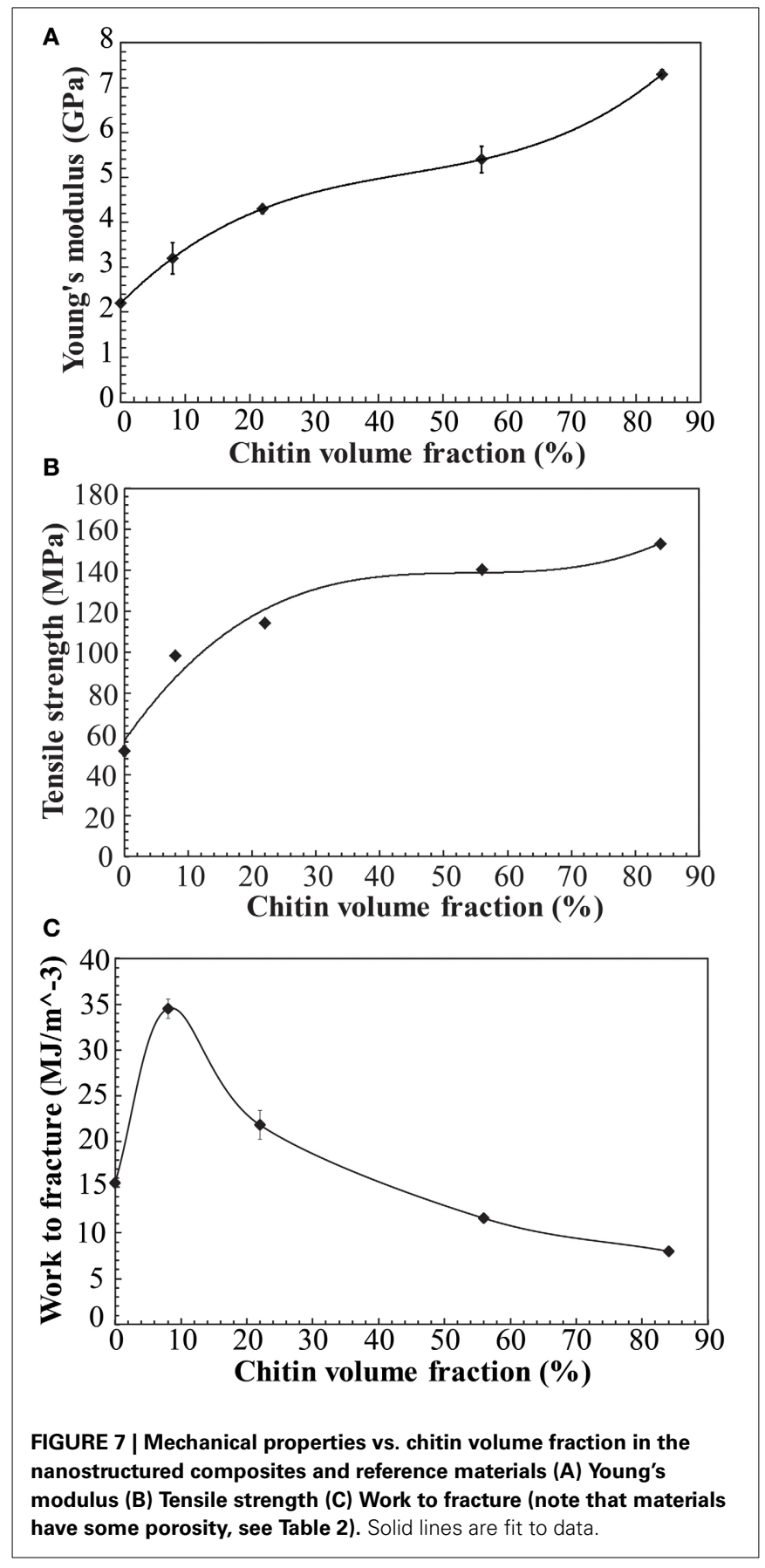

composite will be lower than rule of mixture predictions, as has been demonstrated for composites based on cellulose nanofibers and epoxy (Ansari et al., 2014). One may thus speculate that chitin-chitosan interfacial interaction at molecular scale decreases the density of sites for moisture sorption. With nanoscale fibrils, the specific surface area is very large and interface effects are therefore very strong. Although hygromechanical or thermomechanical strains may influence sorption (Autran et al., 2002; Wan et al., 2005), such effects have not been considered. One reason is that steady-state conditions are reached fairly rapidly in thin films.
Table 4 | Moisture content of the nanostructured composites and reference materials at $\mathbf{5 0}$ and $\mathbf{9 0 \%} \mathrm{RH}$.

\begin{tabular}{lccccc}
\hline Sample description (chitin vol.\%) & 0 & 8 & 22 & 56 & 84 \\
Moisture content at 50\% RH & 15.6 & 8.4 & 8.2 & 7.4 & 10.6 \\
Moisture content at 90\%RH & 34 & 16.8 & 18.1 & 19.7 & 22.9
\end{tabular}

Note that the $84 \mathrm{vol}$ \% composition is a neat chitin nanofiber membrane.

The current data confirm that chitin nanofiber composites show much better mechanical properties compared to chitosan reinforced with chitin whiskers (Sriupayo et al., 2005; Shelma et al., 2008), and also somewhat better properties compared to chitosan composites based on cellulose nanofibers (Fernandes et al., 2010). The deformation mechanisms have been clarified. Compared to the previously reported deacetylated chitin nanofiber-chitosan composites (Ifuku et al., 2013), the present data combine similar strength with the added advantage of high ductility and work to fracture. Chitin nanofibers were not strongly deacetylated as in the study by Fan et al. (2012), where the chitin nanofiber surface was deacetylated to chitosan. The toughness data of the chitin/chitosan composites improve our understanding on the importance of chitin dispersion and chitin-chitosan interaction. The chitin-chitosan-acetic acid combination is also interesting. The work to fracture is similar or slightly better than that of nanostructured composites based on cellulose nanofibers (Sehaqui et al., 2011) (maximum work to fracture $\approx 28 \mathrm{MJ} / \mathrm{m}^{3}$ ). The cellulose nanofibers provide higher strength and modulus, most likely due to better intrinsic strength, stronger interfibril interaction and lower porosity. One may also note that the chitin crystal has lower intrinsic modulus (Ogawa et al., 2011a,b) than cellulose (Sakurada et al., 1964).

\section{DYNAMIC MECHANICAL PROPERTIES}

DMA properties of the nanostructured composites are presented in Figure 8. Previously, Ogura et al studied cast chitin films obtained by dissolution and regeneration(Ogura et al., 1980). It was concluded that chitin degrades thermally prior to its glass transition. In the same study, dry chitosan was reported to show a $\mathrm{Tg}$ of around $140^{\circ} \mathrm{C}$. In Figure $8 \mathrm{~A}$, the thermal stability of chitin network materials is apparent. A gradual decrease of storage modulus with chitin volume fraction and temperature is observed. This is expected, since the chitin nanofiber has much higher modulus than chitosan. The difference in storage modulus between the porous chitin membrane ( $84 \%$ by vol. of chitin) and the chitin/chitosan nanocomposite ( $56 \%$ by volume of chitin) is very small. For chitosan ( $0 \%$ by volume chitin) a softening is observed around $141^{\circ} \mathrm{C}$. At about $219^{\circ} \mathrm{C}$, the chitosan modulus starts to increase, and this indicates thermal degradation and associated cross-linking reactions. This temperature region is associated with elimination of acetamide and amine groups (Kim et al., 1994). From Figure 8B, chitosan shows major tan delta peaks at 188 and $283^{\circ} \mathrm{C}$. The $188^{\circ} \mathrm{C}$ peak is probably associated with $\mathrm{Tg}$. This seems slightly higher than reported in the study by Ogura et al. (1980), but moisture content or the compositional differences between chitosans may explain the differences. In the composites, chitosan transitions are suppressed by the chitin network. 


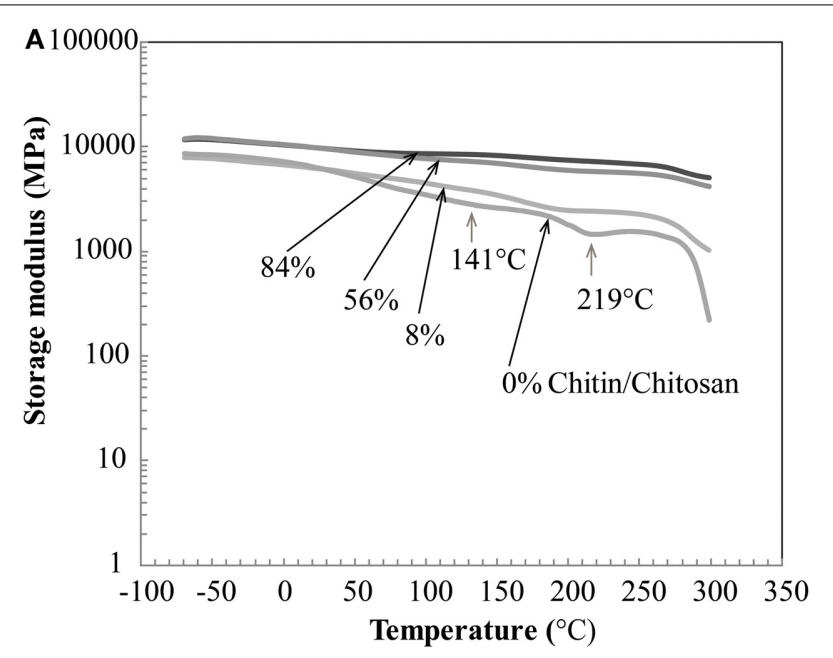

B

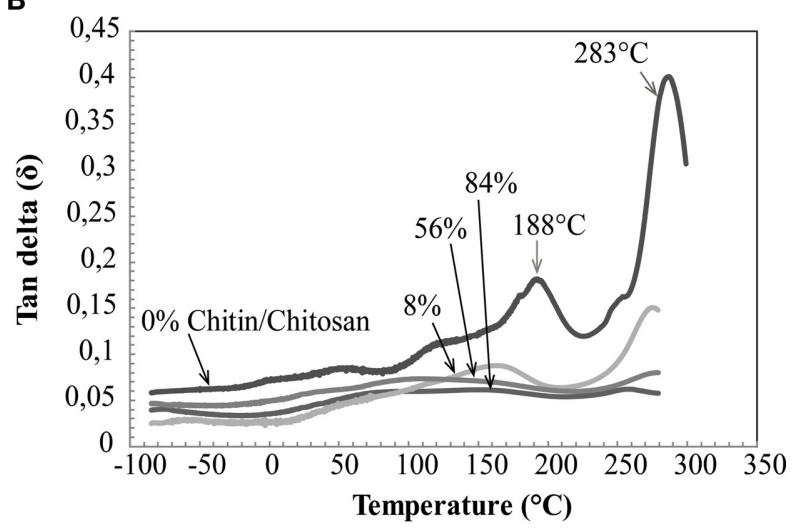

FIGURE 8 | DMA properties of nanostructured chitin composites (56, $8 \%$ chitin by volume), neat chitin membrane ( $84 \%$ chitin by volume) and neat chitosan ( $0 \%$ chitin). (A) Storage modulus vs. temperature and, (B) tan delta vs. temperature.

\section{CONCLUSIONS}

Nanostructured chitin-chitosan nanocomposites completely based on crustacean chitin were prepared. In the context of chitin nanocomposites, the present materials showed a unique combination of modulus, strength and strain-to-failure so that the work to fracture (area under stress-strain curve) was as high as $35 \mathrm{MJ} / \mathrm{m}^{3}$ at a chitin volume fraction of $8 \%$. Also, at very high chitin content (56 vol\%), the nanocomposites showed considerable strength, $140 \mathrm{MPa}$, and strain to failure, $11 \%$. The high strain-to-failure in the nanocomposites is due to reorientation, slippage and straightening of chitin nanofibers in the ductile chitosan matrix. Combined with the small diameter of the chitin and the favorable chitin-chitosan interface interaction, these factors delay formation of microcracks to very high strain. The favorable interface structure is further supported by the observation that moisture sorption of the composites is lower than for either neat chitosan or neat chitin membranes. Most likely, the original moisture sorption sites at the chitin nanofiber surface are no longer available due to strong molecular chitin-chitosan interactions.
The nanostructured material characteristics were confirmed by microscopy. The nanoscale dimensions of the chitin nanofibers prepared in the present study, as well as the low protein content was confirmed. The largest agglomerates in the materials were in the form of a low fraction of fibrous chitin bundles with a diameter of around $100 \mathrm{~nm}$. The rest of the chitin nanofibers showed a diameter at the scale of $10 \mathrm{~nm}$ or less. Colloidal mixtures of chitin nanofibers and dissolved chitosan showed high transparency and good mixing behavior, much better than for the individual components by themselves, and this is essential. Chitin-chitosan repulsion in the colloidal state was confirmed as the main dispersion mechanism. The good colloidal dispersion has favorable effects on chitin nanofiber distribution in the solid material. The nanofibers are well dispersed in the form of curved semi-flexible nanofibers in a chitosan matrix. Fracture surfaces indicate a layered chitin nanofiber structure, and to some extent, flocs are formed as chitin concentration is increased during drying.

Food industry waste in the form of exoskeletons from crab, shrimp, and lobster has potential use in nanostructured chitin/chitosan films of high ductility and strength. In terms of mechanical properties, chitin nanofibers appear to provide better reinforcement effects than chitin nanocrystals due to higher chitin content and the nanofiber network structure. Scientifically, continued focus should be on understanding extraction mechanisms for the nanofibers as well as interface interaction mechanisms in materials containing chitin, chitosan and corresponding counterions. The relevance of published studies on cellulose nanofibers is apparent, and can provide inspiration in future efforts on chitin nanomaterials. Smaller chitin nanofiber diameter, preserved chitin molar mass and tailored chitin-chitosan charge interactions would lead to better chitin dispersion. This is likely to result in high chitin content nanocomposites of even higher toughness than in the present study.

\section{ACKNOWLEDGMENTS}

The authors acknowledge financial support and collaboration benefits through the Carbomat project funded by Formas. Professor Lars Wågberg generously provided access to the QCM and DLS equipment and model surface procedures developed in his laboratory. Dr. Andreas Fall is acknowledged for his kind help with DLS data interpretation.

\section{REFERENCES}

Ansari, F., Galland, S., Johansson, M., Plummer, C. J., and Berglund, L. A. (2014). Cellulose nanofiber network for moisture stable, strong and ductile biocomposites and increased epoxy curing rate. Compos. A 63, 35-44. doi: 10.1016/j.compositesa.2014.03.017

Autran, M., Pauliard, R., Gautier, L., Mortaigne, B., Mazeas, F., and Davies, P. (2002). Influence of mechanical stresses on the hydrolytic aging of standard and low styrene unsaturated polyester composites. J. Appl. Polym. Sci. 84, 2185-2195. doi: 10.1002/app.10419

Bartnicki-Garcia, S. (1968). Cell wall chemistry, morphogenesis, and taxonomy of fungi. Ann. Rev. Microbiol. 22, 87-108. doi: 10.1146/annurev.mi.22.100168. 000511

Carlström, D. (1957). The crystal structure of $\alpha$-chitin (poly-N-acetyl-Dglucosamine). J. Biophys. Biochem. Cytol. 3, 669-683. doi: 10.1083/jcb. 3.5.669 
Chambi, H. N. M., and Grosso, C. R. F. (2011). Mechanical and water vapor permeability properties of biodegradables films based on methylcellulose, glucomannan, pectin and gelatin. Food Sci. Technol. (Campinas) 31, 739-746. doi: 10.1590/S0101-20612011000300029

Chen, C., Li, D., Hu, Q., and Wang, R. (2014). Properties of polymethyl methacrylate-based nanocomposites: reinforced with ultra-long chitin nanofiber extracted from crab shells. Mater. Des. 56, 1049-1056. doi: 10.1016/j. matdes.2013.11.057

Das, P., Heuser, T., Wolf, A., Zhu, B., Demco, D. E., Ifuku, S., et al. (2012). Tough and catalytically active hybrid biofibers wet-spun from nanochitin hydrogels. Biomacromolecules 13, 4205-4212. doi: 10.1021/bm3014796

Fall, A. (2013). Colloidal Interactions and Orientation of Nanocellulose Particles. Ph.D. thesis, Fiber and Polymer Technology, Royal Institute of Technology, DiVA (TRITA-CHE Report 2013:47).

Fall, A. B., Lindström, S. B., Sundman, O., Ödberg, L., and Wågberg, L. (2011). Colloidal stability of aqueous nanofibrillated cellulose dispersions. Langmuir 27, 11332-11338. doi: 10.1021/la201947x

Fan, Y., Fukuzumi, H., Saito, T., and Isogai, A. (2012). Comparative characterization of aqueous dispersions and cast films of different chitin nanowhiskers/nanofibers. Int. J. Biol. Macromol. 50, 69-76. doi: 10.1016/j. ijbiomac.2011.09.026

Fernandes, S., Freire, C. S., Silvestre, A. J., Pascoal Neto, C., Gandini, A., Berglund, L. A., et al. (2010). Transparent chitosan films reinforced with a high content of nanofibrillated cellulose. Carbohydr. Polym. 81, 394-401. doi: 10.1016/j.carbpol.2010.02.037

Gopalan Nair, K., and Dufresne, A. (2003). Crab shell chitin whisker reinforced natural rubber nanocomposites. 2. Mechanical behavior. Biomacromolecules 4, 666-674. doi: 10.1021/bm0201284

Henriksson, M., Berglund, L. A., Isaksson, P., Lindström, T., and Nishino, T. (2008). Cellulose nanopaper structures of high toughness. Biomacromolecules 9, 1579-1585. doi: 10.1021/bm800038n

Henriksson, M., Henriksson, G., Berglund, L., and Lindström, T. (2007). An environmentally friendly method for enzyme-assisted preparation of microfibrillated cellulose (MFC) nanofibers. Eur. Polym. J. 43, 3434-3441. doi: 10.1016/j.eurpolymj.2007.05.038

Ifuku, S., Ikuta, A., Egusa, M., Kaminaka, H., Izawa, H., Morimoto, M., et al. (2013). Preparation of high-strength transparent chitosan film reinforced with surface-deacetylated chitin nanofibers. Carbohydr. Polym. 98, 1198-1202. doi: 10.1016/j.carbpol.2013.07.033

Ifuku, S., Nogi, M., Yoshioka, M., Morimoto, M., Yano, H., and Saimoto, H. (2010). Fibrillation of dried chitin into $10-20 \mathrm{~nm}$ nanofibers by a simple grinding method under acidic conditions. Carbohydr. Polym. 81, 134-139. doi: 10.1016/j.carbpol.2010.02.006

Ji, Y.-L., Wolfe, P. S., Rodriguez, I. A., and Bowlin, G. L. (2012). Preparation of chitin nanofibril/polycaprolactone nanocomposite from a nonaqueous medium suspension. Carbohydr. Polym. 87, 2313-2319. doi: 10.1016/j.carbpol.2011. 10.066

Kienzle-Sterzer, C. A., Rodriguez-Sanchez, D., and Rha, C. (1982). Mechanical properties of chitosan films: effect of solvent acid. Die Makromolekul. Chem. 183, 1353-1359. doi: 10.1002/macp.1982.021830528

Kim, S. S., Kim, S. J., Moon, Y. D., and Lee, Y. M. (1994). Thermal characteristics of chitin and hydroxypropyl chitin. Polymer 35, 3212-3216. doi: 10.1016/00323861(94)90124-4

Lee, Y. M., Kimt, S. H., and Kimt, S. J. (1996). Preparation and characteristics of $\beta$-chitin and poly (vinyl alcohol) blend. Polymer 37, 5897-5905. doi: 10.1016/S0032-3861(96)00449-1

Marx, K. A. (2003). Quartz crystal microbalance: a useful tool for studying thin polymer films and complex biomolecular systems at the solution-surface interface. Biomacromolecules 4, 1099-1120. doi: 10.1021/bm020116i

Mathew, A. P., Laborie, M.-P. G., and Oksman, K. (2009). Cross-linked chitosan/chitin crystal nanocomposites with improved permeation selectivity and $\mathrm{pH}$ stability. Biomacromolecules 10, 1627-1632. doi: 10.1021/bm90 02199

Mima, S., Miya, M., Iwamoto, R., and Yoshikawa, S. (1983). Highly deacetylated chitosan and its properties. J. Appl. Polym. Sci. 28, 1909-1917. doi: 10.1002/app. 1983.070280607

Murakami, K., Aoki, H., Nakamura, S., Nakamura, S., Takikawa, M., Hanzawa, M., et al. (2010). Hydrogel blends of chitin/chitosan, fucoidan and alginate as healing-impaired wound dressings. Biomaterials 31, 83-90. doi: 10.1016/j. biomaterials.2009.09.031

Mushi, N. E., Butchosa, N., Salajkova, M., Zhou, Q., and Berglund, L. A. (2014a). Nanostructured membranes based on native chitin nanofibers prepared by mild process. Carbohydr. Polym. 112, 255-263. doi: 10.1016/j.carbpol.20 14.05.038

Mushi, N. E., Butchosa, N., Zhou, Q., and Berglund, L. A. (2014b). Nanopaper membranes from chitin-protein composite nanofibers-structure and mechanical properties. J. Appl. Polym. Sci. 131, 40121-40130. doi: 10.1002/ app.40121

Muzzarelli, R. A., Boudrant, J., Meyer, D., Manno, N., DeMarchis, M., and Paoletti, M. G. (2012). Current views on fungal chitin/chitosan, human chitinases, food preservation, glucans, pectins and inulin: a tribute to Henri Braconnot, precursor of the carbohydrate polymers science, on the chitin bicentennial. Carbohydr Polym. 87, 995-1012. doi: 10.1016/j.carbpol.2011.09.063

Neville, A. (1967). Chitin orientation in cuticle and its control. Adv. Insect Physiol. 4, 213-286. doi: 10.1016/S0065-2806(08)60209-X

Ogawa, Y., Hori, R., Kim, U.-J., and Wada, M. (2011a). Elastic modulus in the crystalline region and the thermal expansion coefficients of $\alpha$-chitin determined using synchrotron radiated X-ray diffraction. Carbohydr. Polym. 83, 1213-1217. doi: 10.1016/j.carbpol.2010.09.025

Ogawa, Y., Kimura, S., and Wada, M. (2011b). Electron diffraction and highresolution imaging on highly-crystalline $\beta$-chitin microfibril. J. Struct. Biol. 176, 83-90. doi: 10.1016/j.jsb.2011.07.001

Ogura, K., Kanamoto, T., Itoh, M., Miyashiro, H., and Tanaka, K. (1980). Dynamic mechanical behavior of chitin and chitosan. Polym. Bull. 2, 301-304. doi: 10.1007/BF00266704

Park, S. J., Lee, K. Y., Ha, W. S., and Park, S. Y. (1999). Structural changes and their effect on mechanical properties of silk fibroin/chitosan blends. J. Appl. Polym. Sci. 74, 2571-2575. doi: 10.1002/(SICI)1097-4628(19991209)74:11< 2571::AID-APP2>3.0.CO;2-A

Park, S., Marsh, K., and Rhim, J. (2002). Characteristics of different molecular weight chitosan films affected by the type of organic solvents. J. Food Sci. 67, 194-197. doi: 10.1111/j.1365-2621.2002.tb11382.x

Qi, Z.-D., Fan, Y., Saito, T., Fukuzumi, H., Tsutsumi, Y., and Isogai, A. (2013). Improvement of nanofibrillation efficiency of $\alpha$-chitin in water by selecting acid used for surface cationisation. RSC $A d v$. 3, 2613-2619. doi: 10.1039/c2ra $22271 \mathrm{j}$

Raabe, D., Romano, P., Sachs, C., Fabritius, H., Al-Sawalmih, A., Yi, S.-B., et al. (2006). Microstructure and crystallographic texture of the chitinprotein network in the biological composite material of the exoskeleton of the lobster Homarus americanus. Mater. Sci. Eng. A 421, 143-153. doi: 10.1016/j.msea.2005.09.115

Raabe, D., Sachs, C., and Romano, P. (2005). The crustacean exoskeleton as an example of a structurally and mechanically graded biological nanocomposite material. Acta Mater. 53, 4281-4292. doi: 10.1016/j.actamat.2005. 05.027

Sakurada, I., Ito, T., and Nakamae, K. (1964). Elastic moduli of polymer crystals for the chain axial direction. Die Makromolekul. Chem. 75, 1-10. doi: 10.1002/macp.1964.020750101

Sauerbrey, G. (1959). Verwendung von Schwingquarzen zur Wägung dünner Schichten und zur Mikrowägung. Zeitschrift für Physik 155, 206-222. doi: 10.1007/BF01337937

Sehaqui, H., Liu, A., Zhou, Q., and Berglund, L. A. (2010). Fast preparation procedure for large, flat cellulose and cellulose/inorganic nanopaper structures. Biomacromolecules 11, 2195-2198. doi: 10.1021/bm100490s

Sehaqui, H., Mushi, N. E., Morimune, S., Salajkova, M., Nishino, T., and Berglund, L. A. (2012). Cellulose nanofiber orientation in nanopaper and nanocomposites by cold drawing. ACS Appl. Mater. Interfaces 4, 1043-1049. doi: 10.1021/am2016766

Sehaqui, H., Zhou, Q., and Berglund, L. A. (2011). Nanostructured biocomposites of high toughness - a wood cellulose nanofiber network in ductile hydroxyethylcellulose matrix. Soft Matter 7, 7342-7350. doi: 10.1039/clsm $05325 \mathrm{f}$

Shelma, R., Paul, W., and Sharma, C. P. (2008). Chitin nanofibre reinforced thin chitosan films for Wound healing application. Trends Biomater. Artif. Organs 22, 111-115. Available online at: http://www.biomaterials.org.in/ojs/index.php/ tibao/article/view/335 
Sriupayo, J., Supaphol, P., Blackwell, J., and Rujiravanit, R. (2005). Preparation and characterization of $\alpha$-chitin whisker-reinforced chitosan nanocomposite films with or without heat treatment. Carbohydr. Polym. 62, 130-136. doi: 10.1016/j.carbpol.2005.07.013

Svagan, A. J., Azizi Samir, M. A., and Berglund, L. A. (2007). Biomimetic polysaccharide nanocomposites of high cellulose content and high toughness. Biomacromolecules 8, 2556-2563. doi: 10.1021/bm0703160

Wan, Y., Wang, Y., Huang, Y., He, B., and Han, K. (2005). Hygrothermal aging behaviour of VARTMed three-dimensional braided carbon-epoxy composites under external stresses. Compos. A Appl. S 36, 1102-1109. doi: 10.1016/j.compositesa.2005.01.003

Yamamoto, Y., Nishimura, T., Saito, T., and Kato, T. (2010). CaCO3/chitin-whisker hybrids: formation of $\mathrm{CaCO} 3$ crystals in chitin-based liquid-crystalline suspension. Polym. J. 42, 583-586. doi: 10.1038/pj.2010.32

Yusof, N. L., Wee, A., Lim, L. Y., and Khor, E. (2003). Flexible chitin films as potential wound-dressing materials: wound model studies. J. Biomed. Mater. Res. A 66, 224-232. doi: 10.1002/jbm.a.10545
Conflict of Interest Statement: The authors declare that the research was conducted in the absence of any commercial or financial relationships that could be construed as a potential conflict of interest.

Received: 21 August 2014; accepted: 22 October 2014; published online: 18 November 2014.

Citation: Mushi NE, Utsel S and Berglund LA (2014) Nanostructured biocomposite films of high toughness based on native chitin nanofibers and chitosan. Front. Chem. 2:99. doi: 10.3389/fchem.2014.00099

This article was submitted to Polymer Chemistry, a section of the journal Frontiers in Chemistry.

Copyright (C) 2014 Mushi, Utsel and Berglund. This is an open-access article distributed under the terms of the Creative Commons Attribution License (CC BY). The use, distribution or reproduction in other forums is permitted, provided the original author(s) or licensor are credited and that the original publication in this journal is cited, in accordance with accepted academic practice. No use, distribution or reproduction is permitted which does not comply with these terms. 